\title{
Novel Techniques for Real Time Computing Critical Clearing Time SIME-B and CCS-B
}

\author{
Hung Nguyen Dinh*, Minh Y Nguyen* and Yong Tae Yoon ${ }^{\dagger}$
}

\begin{abstract}
Real time transient stability assessment mainly depends on real-time prediction. Unfortunately, conventional techniques based on offline analysis are too slow and unreliable in complex power systems. Hence, fast and reliable stability prediction methods and simple stability criterions must be developed for real time purposes. In this paper, two new methods for real time determining critical clearing time based on clustering identification are proposed. This article is covering three main sections: (i) clustering generators and recognizing critical group; (ii) replacing the multi-machine system by a two-machine dynamic equivalent and eventually, to a one-machineinfinite-bus system; (iii) presenting a new method to predict post-fault trajectory and two simple algorithms for calculating critical clearing time, respectively established upon two different transient stability criterions. The performance is expected to figure out critical clearing time within $100 \mathrm{~ms}-$ $150 \mathrm{~ms}$ and with an acceptable accuracy.
\end{abstract}

Keywords: Critical clearing time, CCS-B, Clustering identification, Post-fault trajectory, Real time computing, SIME-B, Transient stability

\section{Introduction}

Power system stability has been recognized as an important problem for secure system operation since the1920s [1,2]. Many major blackouts caused by power system instability have illustrated the importance of this phenomenon [3]. Transient instability has been the dominant stability problem on most systems, and has been the focus of much of the industry's attention concerning system stability.

Large-disturbance rotor angle stability or transient stability is concerned with the ability of the power system to maintain synchronism when subjected to a severe disturbance, such as a short circuit on a transmission line. Transient stability depends on both the initial operating state of the system and the severity of the disturbance. Instability is usually in the form of aperiodic angular separation due to insufficient synchronizing torque, manifesting as first swing instability. Instability that may result occurs in the form of increasing angular swings of some generators leading to their loss of synchronism with other generators. The basic factor is how the power outputs of synchronous machines vary as their rotor angles change. If the system is perturbed, the equilibrium between the input mechanical torque and the output electromagnetic torque of each generator is upset, resulting in acceleration or deceleration of the rotors of the machines according to the laws of motion of a rotating body. The power-angle

$\dagger$ Corresponding Author: Dept. of Electrical Engineering and Computer Science, Seoul National University, Korea. (ytyoon@ee.snu.ac.kr)

* Dept. of Electrical Engineering and Computer Science, Seoul National University, Korea. (hunghtd@snu.ac.kr, minhy2801@gmail. com)

Received: January 16, 2012; Accepted: September 10, 2012 relationship is highly nonlinear. Beyond a certain limit, an increase in angular separation is accompanied by a decrease in power transfer such that the angular separation is increased further [4].

The dimensions of power network become larger with the development of interconnected local networks. As power systems have evolved through continuing growth in interconnections, use of new technologies and controls, and the increased operation in highly stressed conditions, different forms of system instability have emerged. Hence, a fast and accurate transient stability prediction method is necessary to start up emergency control ensuring security operations of the power grid [5].

There are many transient stability assessment techniques have been proposed such as time-domain (T-D) simulation method [6], transient energy function method [7] and hybrid method [8]. However, contemporary techniques are not fast enough for real time stability assessment. The two methods presented following will fill the need of emergency control in power systems. To reach this objective, the proposed methods have called upon one assumption; that is there are no delays in data collecting from PMUs. This means necessary information is available immediately after the fact.

\section{Clustering Identification}

Mania Pavella et al. pointed out that, like all OneMachine-Infinity-Bus (OMIB)-based methods, SIngle Machine Equivalent (SIME) relies on the following two propositions [9]:

Proposition 1: However complex, the mechanism of loss 
of synchronism in a power system originates from the irrevocable separation of its machines into two groups: one composed of the "critical machines" (CMs, subscript C), which are responsible of the loss of synchronism, the other of the "non-critical machines" (NMs, subscript N).

Proposition 2: The stability properties of an OMIB may be inferred from Equal Area Criterion (EAC) constructed for this OMIB.

Thanks for phasor measurement units (PMUs) presence, the dynamic progress of disturbed power systems can be monitor in real time. Measured data from PMUs then are used to analysis to recognize groups of generators with similar characters or coherent generator groups. In [5], a method to define coherent groups is proposed: when $t \in[0, \tau]$, if the relative power angles of generators $\mathrm{i}$ and $\mathrm{j}$ do not exceed a given criterion $\varepsilon_{0}>0$ at any time, then the two generators can be defined as coherent about the time domain $\tau$, that is

$$
\max _{t \in[0, \tau]}\left|\Delta \delta_{i}(t)-\Delta \delta_{j}(t)\right| \leq \varepsilon_{0}
$$

Usually $\varepsilon_{0}$ can be chosen as $5^{0} \leq \varepsilon_{0} \leq 10^{\circ}$ and $\mathrm{t}$, for real time calculation, must be limited then $30 \mathrm{~ms} \leqslant \mathrm{t} \leqslant 50 \mathrm{~ms}$ with the rate of PMUs is $10 \mathrm{~ms}-20 \mathrm{~ms}$. But simulations show that at the beginning of the disturbances the relative power angles of various generators are not obvious. To get the coherent information faster and more precisely, the first and second derivative of the power angle with respect to time (angular velocity and angular acceleration) can be used as specimens to calculate their space distances. All the $\mathrm{n}$ generators can be grouped into several coherent generator groups [5] by using the following index:

$$
d_{i j}(k)=\frac{d_{\omega i j}(k)}{\sum_{k=1}^{n_{o}} d_{\omega i j}(k)} \text { or } d_{i j}(k)=\frac{d_{\dot{\omega} i j}(k)}{\sum_{k=1}^{n_{o}} d_{\dot{\omega} i j}(k)}
$$

where

$$
\begin{aligned}
& n_{0}=n(n-1) / 2 \\
& d_{\omega i j}(k)=\sqrt{\sum_{k=1}^{K}\left(\Delta \omega_{i}(t)-\Delta \omega_{j}(t)\right)^{2}} \\
& d_{\dot{\omega} i j}(k)=\sqrt{\sum_{k=1}^{K}\left(\Delta \dot{\omega}_{i}(t)-\Delta \dot{\omega}_{j}(t)\right)^{2}}
\end{aligned}
$$

$d_{i j}$ represents the similarity of output trajectory of various generators, if $d_{i j}$ is less than a certain value $\varepsilon$, it means that the dynamic behaviors of the two generators are similar which can be regarded as a coherent generator group, otherwise they belong to different groups. Following the above procedure, $\mathrm{CMs}$ and $\mathrm{NMs}$ are identified. However, the main drawback of the grouping generators is the simplicity of the two-machine (or, one- machine to infinite bus) equivalent which can only approximate an actual multi-machine, multi-bus interconnected electric power system, with lossy components, nonlinearities, high-speed exciters, controllers and stabilizers, regulators, and reactive compensators, having an overall considerable contribution to the transient and dynamic behavior of the system. Since the above contribution is normally beneficial to system stability, the direct methods can only claim a conservative estimation. With the above conditions, any additional simplification introduced in the analysis, or in the computational procedure will further weaken the overall accuracy of the results [12]. Moreover, the performance of grouping generators depends on the value $\varepsilon$.

\section{Network Reduction}

\subsection{The multi-machine model}

In the classical simplified model of an n-machine power system, each generator is represented electrically as a constant voltage source behind its transient reactance and each load as constant impedance. The motion of the $\mathrm{i}^{\text {th }}$ machine is accordingly described by:

$$
\dot{\delta}_{\mathrm{i}}=\omega_{\mathrm{i}} ; \mathrm{M}_{\mathrm{i}} \dot{\omega}_{\mathrm{i}}=\mathrm{P}_{\mathrm{mi}}-\mathrm{P}_{\mathrm{ei}}=\mathrm{P}_{\mathrm{ai}}, \mathrm{i}=1 \ldots, \mathrm{n}
$$

where

$$
\begin{aligned}
& \delta_{\mathrm{i}} \text { :rotor angle } \\
& \omega_{\mathrm{i}}: \text { rotor speed } \\
& \mathrm{M}_{\mathrm{i}}: \text { inertia coefficient } \\
& \mathrm{P}_{\mathrm{mi}}: \text { mechanical input power } \\
& \mathrm{P}_{\mathrm{ei}}: \text { electrical output power } \\
& \mathrm{P}_{\mathrm{ai}}: \text { accelerating power }
\end{aligned}
$$

\subsection{The equivalent two-machine model}

The OMIB transformation results from the decomposition of the system machines into two groups, the aggregation of these latter into their corresponding center of angle (COA). Denoting by $\delta_{C}(t)$ the COA of the group of CMs, one writes:

$$
\begin{array}{ll} 
& \delta_{C}(t) \stackrel{\Delta}{=} M_{C}^{-1} \sum_{k \in C} M_{k} \delta_{k}(t) \\
\text { Similarly: } \quad & \delta_{N}(t) \stackrel{\Delta}{=} M_{N}^{-1} \sum_{j \in N} M_{j} \delta_{j}(t)
\end{array}
$$

In the above formulas: $M_{C}=\sum_{k \in C} M_{k} ; M_{N}=\sum_{j \in N} M_{j}$

\subsection{The equivalent OMIB model}

The expressions of corresponding OMIB parameters $\delta, \omega, M, P_{m}, P_{e}, P_{a}$ are derived as follows. 
a) Define the rotor angle of the corresponding OMIB by the transformation

$$
\delta(t) \stackrel{\Delta}{=} \delta_{C}(t)-\delta_{N}(t)
$$

The corresponding OMIB rotor speed is expressed by

$$
\omega(t) \stackrel{\Delta}{=} \omega_{C}(t)-\omega_{N}(t)
$$

where: $\omega_{C}(t)=M_{C}^{-1} \sum_{k \in C} M_{k} \omega_{k}(t) ; \omega_{N}(t)=M_{N}^{-1} \sum_{j \in N} M_{j} \omega_{j}(t)$

a) Define the equivalent OMIB mechanical power by

$$
P_{m}(t)=M\left(M_{C}^{-1} \sum_{k \in C} P_{m k}-M_{N}^{-1} \sum_{m \in N} M_{m j}\right)
$$

and the resulting OMIB accelerating power by

$$
P_{a}(t)=P_{m}(t)-P_{e}(t)
$$

In the above expressions, $\mathrm{M}$ denotes the equivalent OMIB inertia coefficient

$$
M=\frac{M_{C} M_{N}}{M_{C}+M_{N}}
$$

\section{Proposed Methods}

\subsection{SIME-based method (SIME-B)}

\section{a) SIME revisited}

Literature [9] investigated on SIME. SIME is a transient stability method based on a generalized OMIB. The fundamental difference between the original version of Extended Equal-Area Criterion (EEAC) and SIME is that EEAC relies on a time-invariant OMIB that constructs by assuming the classical simplified machine and network modeling and by "freezing" once and for all the machine rotor angles at $t_{0}$, the initial time of the disturbance inception.

\section{- Equal Area Criterion revisited}

EAC relies on the concept of energy. In short, it states that the stability properties of a contingency scenario may be assessed in terms of the stability margin, defined as the excess of the decelerating over the accelerating area of the OMIB $P_{a}-\delta$ plane:

$$
\eta=A_{d e c}-A_{a c c}
$$

- Conditions of unstable OMIB trajectory

An unstable case corresponds to $\eta<0$, i.e., to

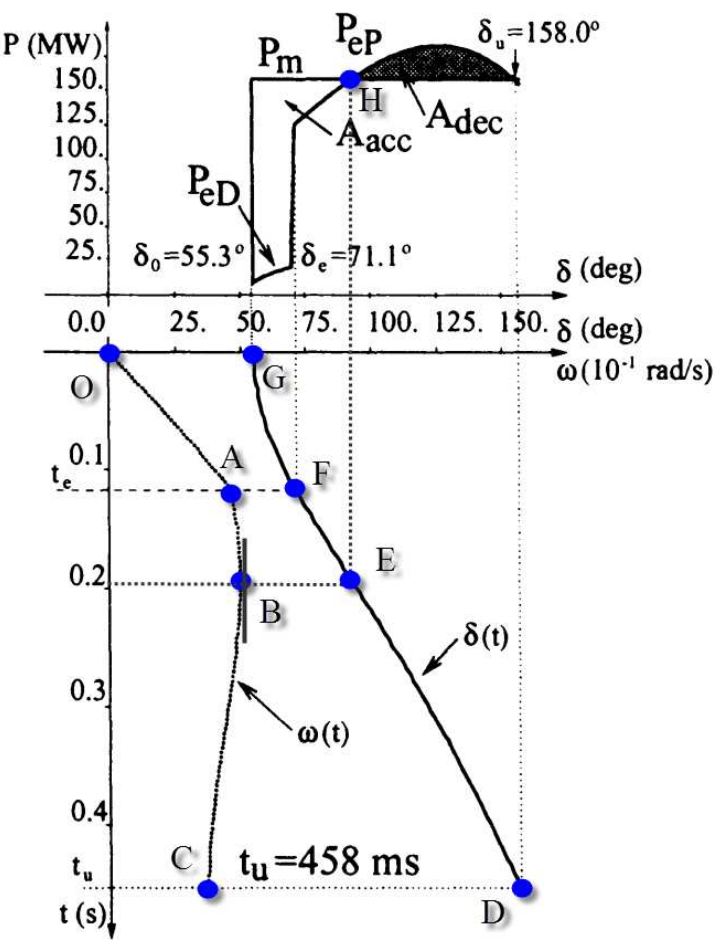

Fig. 1. OMIB $P-\delta$ and T-D representation with $t_{e}=$ $117 \mathrm{~ms}$, unstable case

$A_{d e c}<A_{a c c}$. In such a case, the curve $P_{e P}$ crosses $P_{m}$ or, equivalently, the accelerating power $P_{a}$ passes by zero and continues increasing. From a physical point of view, $P_{a}=0$ takes place at $\delta=\delta_{u}$ and marks the OMIB loss of synchronism. An unstable OMIB trajectory reaches the unstable angle $\delta_{u}$ at time $t_{u}$ as soon as:

$$
P_{a}\left(t_{u}\right)=0, \quad \dot{P}_{a}\left(t_{u}\right)=\left.\frac{d P_{a}}{d t}\right|_{t=t_{u}}>0
$$

- Conditions of stable OMIB trajectory

A stable case corresponds to $\eta>0$, i.e., to $A_{d e c}>A_{a c c}$. Inspection of Figs. 1 or Fig. 2 suggests that in such a case, the acquired kinetic energy is less than the maximum potential energy: $P_{e P}$ stops its excursion at $\delta=\delta_{r}$, before crossing $P_{m}$. Stated otherwise, at $\delta=\delta_{r}, \omega=0$ with $P_{a}<0$, $\delta$ stops increasing then decreases. The above reasoning yields the following statement: a stable OMIB trajectory reaches the return angle $\delta_{r}\left(\delta_{r}=\delta_{u}\right)$ at time $t_{r}$ as soon as

$$
\omega\left(t_{r}\right)=0, \text { with } P_{a}\left(t_{r}\right)<0
$$

\section{b) Proposed method based on SIME (SIME-B)}

SIME-B method is developed based on angular velocity prediction and a new transient stability assessment derived from conditions of stable OMIB trajectory. Furthermore, the criterion is made simpler from an interesting observation. Since the new criterion is quite simple, 


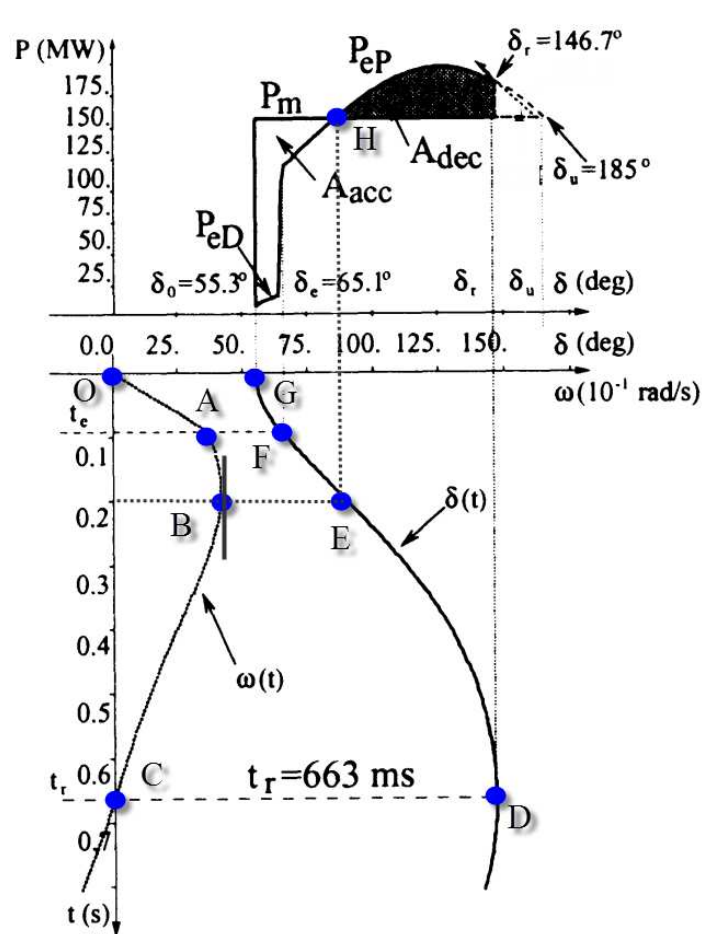

Fig. 2. OMIB $P-\delta$ and T-D representation with $t_{e}=$ $92 m s$, stable case

computation speed is extremely fast and tallies with real time assessment.

\section{- The angular velocity prediction}

Literature [10] first proposed that the generator angular velocity can be obtained by Newton interpolation method and the generator angle in a future time can be calculated by integration of the angular velocity. Since the generator rotor has considerable large inertia, the variation of its angular velocity $\omega$ is a smooth procedure.

In order to determine the critical clearing time CTT, the computation method needed be able to predict the postfault trajectory after fault is cleared. However, that prediction merely based on commencing data directly following fault occurs, normally, contains significant errors. The reasons are the $P_{a}$ curve is not smooth and clearance time $t_{e}$ is still a variable. In this paper, a new method on the basis of the angular velocity prediction successfully addresses that problem. Extrapolating angular velocity with a quadratic function of time is a reasonable choice. Hence, $\mathrm{ABC}$ curve is of the form:

$$
\omega(t)=a t^{2}+b t+c, a<0, t \geq t_{A}
$$

and solving for $\mathrm{a}, \mathrm{b}, \mathrm{c}$ based on $\omega(t)$ values taken at three successive time steps. One of these values is angular velocity at point $\mathrm{A}, t_{A}=t_{e}$, when fault is cleared, and the others at $t_{A} \pm \Delta t, \Delta t=10 \mathrm{~ms}$. The section OA is nearly linear, therefore, assume that $\mathrm{OA}$ is of the form:

$$
\omega(t)=k t, t_{O} \leq t \leq t_{A}
$$

Obviously, when we move A along the line OA, a, b, c must be different. That is the key of the new method and is able to illustrate how the clearance time affects the following predictions.

Moreover, angle of rotor and angular acceleration are of the forms:

$$
\begin{gathered}
\delta(t)=\frac{1}{3} a t^{3}+\frac{1}{2} b t^{2}+c t+d \\
\dot{\omega}(t)=2 a t+b
\end{gathered}
$$

$t \geq t_{A}$

Once $\mathrm{a}, \mathrm{b}$ and $\mathrm{c}$ are determined and commencing data of angle are available, angle curve $\delta(t)$ and angular acceleration curve $\dot{\omega}(t)$ for $t \geq t_{A}$ are resolved as well. Taking three points on angular acceleration curve $\dot{\omega}(t)$ and combining with swing equation:

$$
M \dot{\omega}=P_{a}
$$

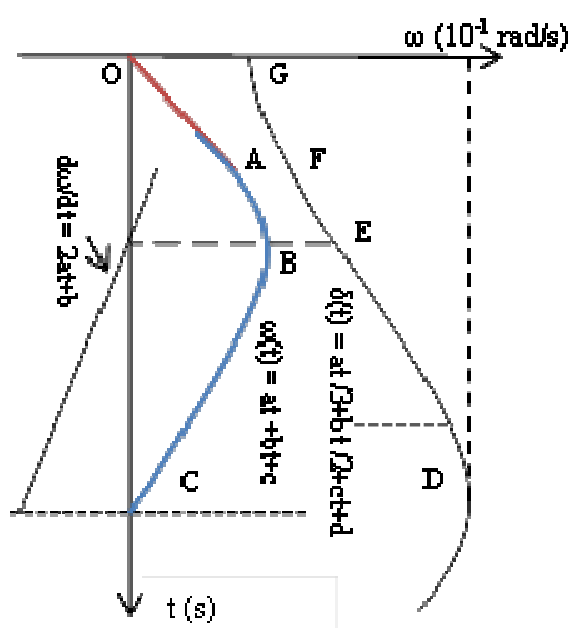

Fig. 3. Post-fault trajectory prediction

corresponding values of $P_{a}$ will be found. Referring these values of $P_{a}$ to angle curve $\delta(t), P_{a}$ curve is defined for $t \geq t_{A}$ :

$$
P_{a}(\delta)=m \delta^{2}+p \delta+q, m<0
$$

In other words, $\mathrm{m}, \mathrm{p}$ and $\mathrm{q}$ are solved. Since sufficient information is attained, either EEAC or SIME method can be applied to compute CCT.

- The new transient stability assessmentand CCT determining algorithm

Firstly, "angle to instability" $\delta_{u}$ can be computed as:

$$
P_{a}\left(\delta_{u}\right)=m \delta_{u}^{2}+p \delta_{u}+q=0
$$




$$
\text { Since } \delta_{u}>\delta_{H}, \delta_{u}=\frac{-p-\sqrt{p^{2}-4 m q}}{2 m}
$$

Similarly, from angular velocity curve $\omega(t), t_{C}$ is found as:

$$
\omega\left(t_{C}\right)=a t_{C}^{2}+b t_{C}+c=0
$$

Then,

$$
t_{C}=\frac{-b-\sqrt{b^{2}-4 a c}}{2 a}
$$

The maximum angle in stable case, $\delta_{D}$, may calculated as:

$$
\delta_{D}=\frac{1}{3} a t_{C}^{3}+\frac{1}{2} b t_{C}^{2}+c t_{C}+d ; t_{D}=t_{C}
$$

The condition of stable trajectory can be restated as: if $\delta_{D}<\delta_{u}$, the system is first-swing stable; if $\delta_{D}>\delta_{u}$, the system is unstable. The statement is valid since the system is stable if the maximum angle $\delta_{D}$ is smaller than "angle to instability" $\delta_{u}$. Based on this claim, a new algorithm to determine CCT is proposed with 4 steps:

i. Initial clearing time conditions: select a value of $t_{A}$ for the first location of point $A$, say $t_{A}=100 \mathrm{~ms}$ after fault occurs

- Depicting OA segment with coefficient $\mathrm{k}$ or building angular velocity curve $\omega(t)$ for $t \leq t_{A}$

- Estimating a, b and c to find angular velocity curve, angle of rotor and angular acceleration curve.

- Predicting $P_{a}$ and determining $\mathrm{m}, \mathrm{p}, \mathrm{q}$

ii. Computing $\delta_{D} \& \delta_{u}$

iii.

- If $\delta_{D}<\delta_{u}$, increasing or move point $\mathrm{A}$ further to point $\mathrm{O}$ along $\mathrm{OA}$ segment and returning to $\mathrm{i}$.

- If $\delta_{D}>\delta_{u}$, decreasing or move point $\mathrm{A}$ closer to point $\mathrm{O}$ along $\mathrm{OA}$ segment and returning to $\mathrm{i}$.

iv. Iteration process is complete if in two successive steps, $\delta_{D}-\delta_{u}$ changes its sign from positive to negative or vice versa. CTT is determined as average of the last two clearing times.

\section{Remarks:}

- The proposed method is faster than the existing SIME due to its simple interpolation and criterions. The difference becomes clearer when the CCT-finding procedures are used repeatedly. The larger number of iterations, the more computationally interesting the proposed method becomes with respect to the existing one

- With respect to the very high non-linear issue, we observe that while the electric power outputs behave in a highly on-linear way particularly at the moment of fault clearance, the angle dynamics and the angle velocity dynamics remain "nice", which allows for an accurate extrapolation using a quadratic function. This phenomenon may be a result of physical characteristics of generator rotor having considerable inertia. Since the electric power outputs are complex and depend on clearance time which is still unknown, the predicting steps and the criterions of the proposed method mainly rely on the angle velocity rather than the electric power outputs. This point also distinguishes the proposed methods from the existing SIME in which the electric power is directly predicted and the criterion is defined via the negative margin energy which depends on the electric power.

\section{- Simplifieralgorithm}

The above algorithm appeals simply to carry out with computers and saves time comparing with the conventional one. Nevertheless, for the sake of real-time computation purposes, it needs to be improved. Notice that in both stable and unstable cases, $t_{B}$ is almost fixed. The reason is the rotor normally has a large inertia. At point $\mathrm{B}$, angular velocity reaches its maximum, $\omega\left(t_{B}\right)=\omega_{\max }$ and angular acceleration equals zero $\dot{\omega}\left(t_{B}\right)=0$. E is the point on angle curve such that $t_{E}=t_{B}$. From swing equation and $\dot{\omega}\left(t_{B}\right)=0$, point $\mathrm{E}$ is corresponding with point $\mathrm{H}$ on $P_{a}$ curve, where $P_{a H}=P_{m}-P_{e}=0$. Relied on these observations, we can assume that point $\mathrm{H}$ and $P_{a}$ curve are unchanged. Then $P_{a}$ curve is defined from the first iteration and kept unchanged through the simulation procedure. Finally, computational intensity reduces by a considerable amount.

Admittedly, the simplifier algorithm is based on the simplifying assumption which would affect the accuracy of the results. However, experiments pointed out that the errors are in the acceptable range. This can be explained as an effect of the inertial delay.

\subsection{Critical condition for synchronism-based method (CCS-B)}

\section{a) Critical condition for synchronism revisited}

In [11], Naoto Yorino et al. proposed a critical condition for synchronism for multi-machine systems. As is given in Fig. 4, the critical trajectory converges to unstable

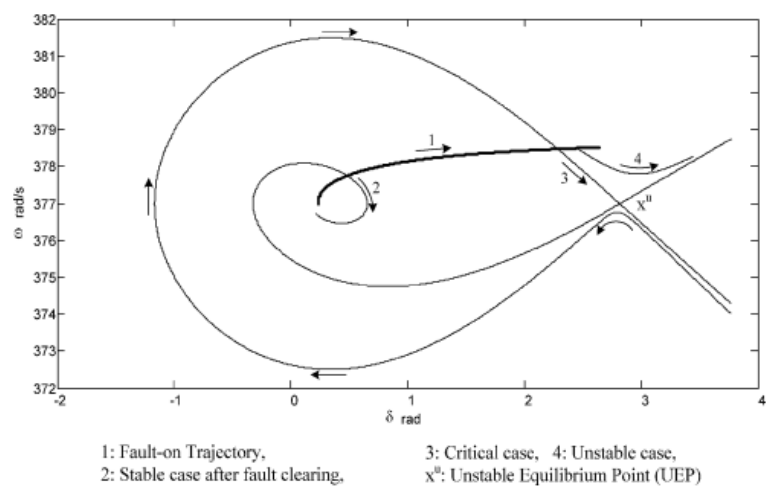

Fig. 4. Trajectories in a phase plane for a single machine to infinite bus system with damping. 
equilibrium point (UEP) for a single machine infinite bus system. In this section, the authors propose critical condition for synchronism which suffices in general multimachine systems. It is known in a single machine case that the synchronizing force disappears when $\frac{\partial P}{\partial \delta}=0$, where $P$ is the synchronizing power. A natural extension of the condition for multi-machine case may be written based on singularity condition of synchronizing force coefficient matrix as follows:

$$
\left[\frac{\partial P}{\partial \delta}\right] \cdot v=0, \text { with } v \neq 0
$$

where $v \in R^{n}$ is the eigenvector corresponding to zero eigenvalue of matrix $\left[\frac{\partial P}{\partial \delta}\right] \in R^{n x n}$, and $\mathrm{n}$ is the number of generators. The authors further intuitively assume a condition that the eigenvector must agree with change direction of $\delta$. That is, the following equation holds with a scalar $k_{S} \in R$ :

$$
v=k_{S} . \dot{\delta}
$$

It will be assumed that the above conditions (22) and (23) hold at a point (the end point) on the critical trajectory. Although it is not the complete proof of the stability condition for dynamic system, the equations represent the stationary conditions for the synchronizing power, or as follows:

$$
\dot{P}=0
$$

Since $P$ is basically a function of the rotor angles of generators, the following equation holds:

$$
\dot{P}=\left[\frac{\partial P}{\partial \delta}\right] \cdot \dot{\delta}=0
$$

The conditions have been derived intuitively by the author and will be used without complete proof for instability.

\section{(a) The complete proof of critical condition for synchronism for the two-machine case}

The two-machine model is obtained after transforming critical machines group $(\mathrm{C})$ and noncritical machines group (N) into two equivalent machines.

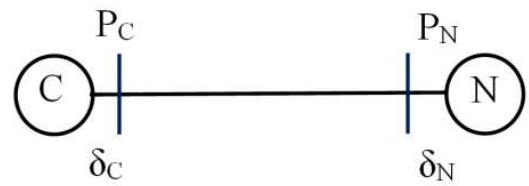

Fig. 5. The two-machine model where $P_{C}, P_{N}$ : electric output of $\mathrm{CMs}$ and NMs, respectively

$\delta_{C}, \delta_{N}$ : electric output rotor angle of CMs and NMs, respectively

We define:

$$
\begin{gathered}
P=\left[\begin{array}{l}
P_{C} \\
P_{N}
\end{array}\right] ; \delta=\left[\begin{array}{l}
\delta_{C} \\
\delta_{N}
\end{array}\right] \\
{\left[\frac{\partial P}{\partial \delta}\right]=\left[\begin{array}{ll}
\frac{\partial P_{C}}{\partial \delta_{C}} & \frac{\partial P_{C}}{\partial \delta_{N}} \\
\frac{\partial P_{N}}{\partial \delta_{C}} & \frac{\partial P_{N}}{\partial \delta_{N}}
\end{array}\right]} \\
\dot{\delta}=\left[\begin{array}{l}
\dot{\delta}_{C} \\
\dot{\delta}_{N}
\end{array}\right]
\end{gathered}
$$

In the vicinity of the end point of the critical trajectory, we can yield:

$$
\dot{\delta}_{C}=k_{\delta} \cdot \dot{\delta}_{N}
$$

where $k_{\delta}$ is calculated by two successive values of angle. From (25), we have:

$$
\dot{P}_{C}=k_{P} \frac{\partial P_{C}}{\partial \delta_{C}}
$$

Swing equation is rewritten as:

$$
M_{C} \ddot{\delta}_{C}=P_{a}
$$

Taking approximation:

$$
M_{C} \ddot{\delta}_{C}=\dot{P}_{a} \cdot \Delta \delta_{C}
$$

Since $P_{a}=P_{m}-P_{e}$ and $P_{m}=$ const then $\dot{P}_{a}=\dot{P}_{e}=\dot{P}_{C}=h$; and $\Delta \delta_{C}=\delta_{C}(t)-\delta_{C}(t=0)=\delta_{C}-$ const then $\ddot{\delta}_{C}=\Delta \ddot{\delta}_{C}$, we have:

$$
M_{C} \cdot \Delta \ddot{\delta}_{C}=h \cdot \Delta \delta_{C}
$$

If $\mathrm{h}<0,(33)$ has real roots of the form:

$$
\Delta \delta_{C}(t)=a_{1} e^{\omega_{n} t}+a_{2} e^{-\omega_{n} t}
$$

where $\omega_{n}=\sqrt{\frac{-h}{M_{C}}}$

The part of $a_{1} e^{\omega_{n} t}$ will go to infinity or the system will be unstable.

Then $\mathrm{h}=0$ is the boundary of stability. 


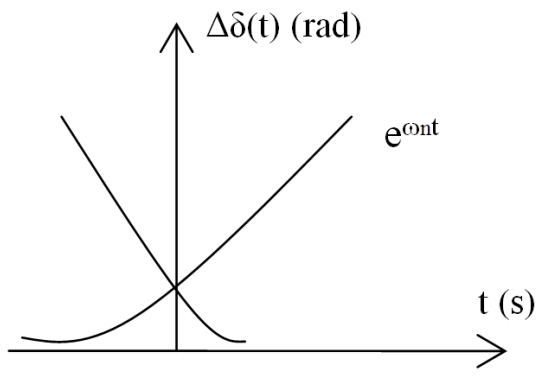

Fig. 6. Unstable case with a negative $h$

\section{c) The proposed CCS-B method}

CCS-B method is alternative one in this paper, which basically relied on behavior of the coefficient $\frac{d P_{C}}{d \delta_{C}}=h$. When $\mathrm{h}$ changes its sign from positive to negative, the system loses synchronism. The main difference between CCS-B method and SIME-B is that in CCS-B, the system is only reduced to a two-machine model. Therefore, the result is more accuracy. Similar to SIME-B, the CCS-B algorithm relies on the 4 following steps:

i. Initial clearing time conditions: select a value of $t_{A}$ for the first location of point $\mathrm{A}$, say $\mathrm{t}_{\mathrm{A}}=100 \mathrm{~ms}$ after fault occurs

- Depicting OA segment with coefficient k or building angular velocity curve $\omega(t)$ for $t \leq t_{A}$

- Estimating a, b and c to find angular velocity curve, angle of rotor and angular acceleration curve.

- Predicting $\frac{d P_{C}}{d \delta_{C}}$

ii. Computing $\delta_{D} \& \delta_{c r i}$, where $\delta_{c r i}$ satisfies

$$
\frac{d P_{C}}{d \delta_{c r i}}=0
$$

iii.

- If $\delta_{D}<\delta_{c r i}$, increasing or move point $\mathrm{A}$ further to point $\mathrm{O}$ along $\mathrm{OA}$ segment and returning to $\mathrm{i}$.

- If $\delta_{D}>\delta_{c r i}$, decreasing or move point A closer to point $\mathrm{O}$ along $\mathrm{OA}$ segment and returning to $\mathrm{i}$.

iv. Iteration process is complete if in two successive steps, $\delta_{D}-\delta_{c r i}$ changes its sign from positive to negative or vice versa. CTT is determined as average of the last two clearing times.

Notice that OMIB model in SIME-B is replaced with CMs model in CSS-B method.

\section{Case Study}

\subsection{Numerical examination for SIME-B method}

In order to illustrate the effectiveness of SIME-B Method, we have carried out numerical examinations using 10-machine, 39-bus New England Test System. The simulation platform used for the proposed approach is the PC with Intel(R), Core(TM)-2 CPU-2.13 GHz with 4 GB of RAM.

Comparing with conventional time domain simulation method, SIME-B method has shown a good performance with small errors around $0.002 \mathrm{~s}-0.005 \mathrm{~s}$ while consuming time is extremely fast, less than $105 \mathrm{~ms}$. For SIME-B simplifier method, the errors are around $0.001 \mathrm{~s}-0.007 \mathrm{~s}$ and the consuming time is less than $93 \mathrm{~ms}$. The estimated CCTs for various fault location have listed in Table 1 below:

Table 1. CCTs of 39-Bus new england test system

\begin{tabular}{c|c|c|c|c|c}
\hline \multirow{2}{*}{$\begin{array}{c}\text { Fault } \\
\text { line }\end{array}$} & \multirow{2}{*}{$\begin{array}{c}\text { CCT by } \\
\text { T-D method } \\
\left(10^{-3}\right)\end{array}$} & \multicolumn{2}{c|}{$\begin{array}{c}\text { CCT by SIME-B } \\
\left(10^{-3} \mathrm{~s}\right)\end{array}$} & \multicolumn{2}{c}{$\begin{array}{c}\text { Computing time of } \\
\text { SIME-B }\left(10^{-3} \mathrm{~s}\right)\end{array}$} \\
\cline { 3 - 6 } & 263 & Full & Simplifier & Full & Simplifier \\
\hline $2-3$ & 268 & 270 & 90 & 80 \\
\hline $4-14$ & 256 & 261 & 259 & 102 & 93 \\
\hline $6-11$ & 237 & 235 & 233 & 105 & 92 \\
\hline $15-16$ & 241 & 244 & 240 & 87 & 80 \\
\hline $23-24$ & 216 & 213 & 218 & 101 & 92 \\
\hline $25-26$ & 216 & 216 & 215 & 97 & 81 \\
\hline
\end{tabular}

\subsection{Test results of CCS-B method}

CCS-B method has been tested on IEEE 7-machine, 57bus system. The results pointed out precise values of CTT with deviations around $0.001 \mathrm{~s}$ and execution time is less than $101 \mathrm{~ms}$. The results are more accuracy than SIME-B method since the system is only reduced to a two-machine system. Computation results are shown in the following table:

Table 2. CCTs of IEEE 57-Bus system

\begin{tabular}{c|c|c|c|c|c}
\hline \multirow{2}{*}{$\begin{array}{c}\text { Fault } \\
\text { line }\end{array}$} & $\begin{array}{c}\text { CCT by T-D } \\
\text { method } \\
\left(10^{-3} \text { s }\right)\end{array}$ & \multicolumn{2}{|c|}{ CCT by $\left(10^{-3}\right.$ s $)$} & \multicolumn{2}{c}{ Computing time $\left(10^{-3}\right.$ s $)$} \\
\cline { 3 - 6 } & 171 & 170 & 168 & 86 & 89 \\
\hline $2-3$ & 241 & 241 & 244 & 92 & 95 \\
\hline $3-4$ & CCS-B & $\begin{array}{c}\text { SIME-B } \\
(\text { Full })\end{array}$ & CCS-B & $\begin{array}{c}\text { SIME-B } \\
(\text { Full })\end{array}$ \\
\hline $3-15$ & 237 & 236 & 234 & 80 & 85 \\
\hline $4-5$ & 306 & 306 & 309 & 101 & 99 \\
\hline $6-5$ & 183 & 184 & 186 & 100 & 98 \\
\hline $6-8$ & 182 & 183 & 182 & 82 & 85 \\
\hline $7-8$ & 189 & 189 & 188 & 85 & 87 \\
\hline
\end{tabular}

Table 3. CCTs of IEEE 118-Bus system

\begin{tabular}{c|c|c|c|c|c}
\hline \multirow{2}{*}{$\begin{array}{c}\text { Fault } \\
\text { line }\end{array}$} & $\begin{array}{c}\text { CCT by } \\
\text { T-D method } \\
\left(10^{-3} \mathrm{~s}\right)\end{array}$ & \multicolumn{2}{|c|}{ CCT by $\left(10^{-3} \mathrm{~s}\right)$} & \multicolumn{2}{c}{$\begin{array}{c}\text { Computing time } \\
\left(10^{-3} \mathrm{~s}\right)\end{array}$} \\
\cline { 3 - 6 } & CCS-B & $\begin{array}{c}\text { SIME-B } \\
(\text { Simplifier })\end{array}$ & CCS-B & $\begin{array}{c}\text { SIME-B } \\
(\text { Simplifier })\end{array}$ \\
\hline $1-3$ & 825 & 820 & 834 & 135 & 136 \\
\hline $15-33$ & 670 & 673 & 678 & 129 & 130 \\
\hline $37-39$ & 586 & 592 & 572 & 126 & 126 \\
\hline $100-103$ & 270 & 277 & 261 & 124 & 120 \\
\hline $105-107$ & 761 & 755 & 758 & 132 & 119 \\
\hline $104-105$ & 1005 & 1010 & 1015 & 131 & 136 \\
\hline
\end{tabular}

When the systems become larger, much time for handling the big data and running the T-D program will 
slow down the CCT determining process. Parallel computing in case of centralized control scheme or distributed computing in case of distributed control scheme should be applied in order to reduce the computing time needed and make the proposed methods becomes even more attractive to real-time applications.

\section{Conclusion}

This paper proposed two new methods for transient stability assessment: SIME-B and CCS-B with introduction of PMUs. CTT is determined in extremely short time, obviously, is eligible for real time analysis. The new model applied for predicting post-fault trajectory based on angular velocity prediction successfully overcomes problem of accelerating power estimation, which is unsmooth. SIME$B$ method can be executed even faster sincethe time angular velocity reaches the maximum, $t_{B}$, is fixed. However, in some cases, a large deviation of $P_{a}$ will lead to inaccurate results. On the other hand, CCS-B seems to be a promising method because of its precision and rapid convergence.

\section{Acknowledgements}

This work is inthe research project (2011T100100152) which has been supported by Korean Electrical Engineering \& Science Research Institute (KESRI) and Korean Institute of Energy Technology Evaluation and Planning (KETEP), which is funded by Ministry of Knowledge Economy (MKE).

\section{References}

[1] C. P. Steinmetz, "Power control and stability of electric generating stations", AIEE Trans., Vol. XXXIX, Part II, pp. 1215-1287, July 1920.[1] C. P. Steinmetz, "Power control and stability of electric generating stations", AIEE Transactions, Vol. XXXIX, Part II, pp. 1215-1287, July 1920.

[2] AIEE Subcommittee on Interconnections and Stability Factors, "First report of power system stability," AIEE Transactions, pp. 51-80, 1926.

[3] G. S. Vassell, "Northeast blackout of 1965", IEEE Power Engineering Review, pp. 4-8, Jan. 1991.

[4] Kundur, P.; Paserba, J.; Ajjarapu, V.; Andersson, G.; Bose, A.; Canizares, C.; Hatziargyriou, N.; Hill, D.; Stankovic, A.; et al. "Definition and classification of power system stability IEEE/CIGRE joint task force on stability terms and definitions", Power Systems, IEEE Transactions, Vol. 19, Issue:3, pp.1387-1401, Aug. 2004.
[5] Yujing Wang; Jilai Yu, "Real Time Transient Stability Prediction of Multi-Machine System Based on Wide Area Measurement", Power and Energy Engineering Conference, 2009, APPEEC 2009, Asia-Pacific, pp. 1-4, March 2009.

[6] Wang Fangzong, Chen Deshu, He Yangzan, “On-line transient stability simulation and assessment of largescale power system", Proceedings of the CSEE, Vol. 13, No. 6, pp. 13-19, 1993.

[7] Fouad A A, Vittal V, "Power system transient stability analysis using the transient energy function method", Prentice Hall, Inc., 1992.

[8] Maria G A, Tang C, Kim J., "Hybrid transient stability analysis", IEEE Transactions on PWRS., Vol. 5, No. 2, pp. 384-393, 1990.

[9] Mania Pavella, Damien Ernst, Daniel Ruiz-Vega, "Transient Stability of Power System A Unified Approach to Assessment and Control", Kluwer Academic Publishers, 2000

[10] M. Takahashi, K. Matsuzawa, M. Sato, et al. "Fast generation shedding equipment based on the observation of swings of generators." IEEE Transactions on Power Systems, Vol. 3, No. 2, pp. 439-446, May 1988.

[11] Yorino, N.; Priyadi, A.; Kakui, H.; Takeshita, M., "A New Method for Obtaining Critical Clearing Time for Transient Stability", Power Systems, IEEE Transactions, Vol. 25, Issue: 3, pp. 1620-1626, Aug. 2010.

[12] Y. Xue, Th. Van Cutsem, M. Ribbens-Pavella, "A simple direct method for fast transient stability assessment of large power systems", IEEE Trans. on Power Systems, Vol. 3, No. 2, May 1988.

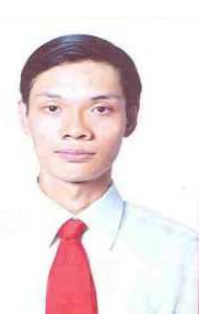

Hung Nguyen Dinh was born in Vietnam on May 06, 1986. He received the B.S. degrees in Electrical Engineering from Hanoi University of Technology, Vietnam, in 2009. Currently, he is pursuingM.S. degree in Electrical Engineering in Seoul National University, Korea. His research interests are in the areas of power system analysis and control, the dynamic behavior of large system and decentralized control.

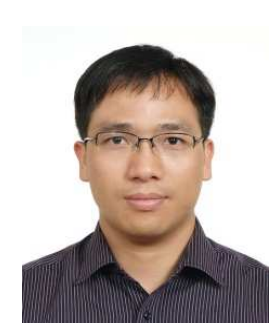

Minh Y Nguyen was born in Vietnam in 1983. He received a B.S. of Electrical Engineering from Hanoi University of Technology, Vietnam in 2006, M.S. of Electrical Engineering from Seoul National University, Korea in 2009. Currently, he is pursuing Doctoral Degree in Dept. of Electrical Engineering and Computer Science, Seoul National University, Korea. His research fields of interest include restructured electric power industry, micro-grid, smart-grid and integration of alternative energy sources. 


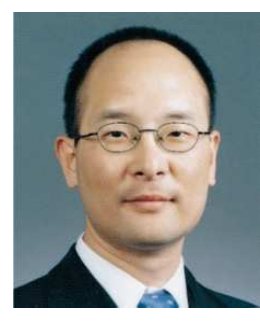

Yong Tae Yoon was born in Korea on April 20, 1971. He received the B.S. degree, M.Eng. and Ph.D. degrees from M.I.T., USA in 1995, 1997, and 2001, respectively. Currently, he is an Associate Professor in the School of Electrical Engineering and Computer Science at Seoul National University, Korea. His special field of interest includes electric power network economics, power system reliability, and the incentive regulation of independent transmission companies. 